were issued to potential new members (43) and renewing members (13) to a meeting in September 2016. Terms of Reference, which were discussed at the meeting and amended, were issued to 54 people in November 2016. Receipt of a signed copy confirmed membership of the group. A total of 44 members confirmed membership. Since September 2016 they have been involved in a range of activities informing palliative care education, research, policy and practice across both the Republic of Ireland and Northern Ireland.

\section{P-229 EXPLORING PALLIATIVE CARE NEEDS: A CONSULTATION WITH YOUNG PEOPLE WITH LIFE-LIMITING CONDITIONS}

${ }^{1}$ Johanna Taylor, ${ }^{2}$ Jan Aldridge. ${ }^{1}$ University of York, York, UK; ${ }^{2}$ Martin House Hospice, Wetherby, UK

\subsection{6/bmjspcare-2017-hospice.254}

Background Paediatric palliative care plays an important role in the lives of children and young people with life-limiting conditions and their families. However, the views of young people are rarely sought when improving these services.

Aims This consultation explored the views and experiences of young people, with an aim to inform clinical guidelines.

Methods Qualitative thematic analysis of data collected from 14 young people (aged 12-18) with a life-limiting condition, who took part in focus groups or interviews. Topics explored were information and communication; care planning; place of care; emotional care; and improving services.

Findings Participants were keen to be involved in care planning, although preferences for involvement and information varied and changed over time. Participants emphasised that quality of care was more important than place of care. Aspects that helped them to feel safe and looked after included: access to specialists with knowledge of their condition; being treated as an individual; carers taking time to meet their personalised needs; and continuity of relationships. Participants described a range of negative feelings related to having a serious health condition. Distraction, avoidance, and talking to others were identified as common strategies to manage these. However, some participants expressed unmet needs for emotional care and felt unable to discuss this with others. Conclusions Providing young people with personalised information and involving them in planning care to the extent they desire can reduce uncertainty about what will happen and when, and minimise disruption to everyday life. This needs to be balanced against their developmental and emotional needs, and the importance to young people of living well rather than focusing on their deteriorating health and end of life.

\section{P-230 'YOU SAID WE DID': LEARNING FROM SERVICE USERS AT SAINT FRANCIS HOSPICE}

Bridget Moss, Jan Scott, Tracie Brennan, Clare McPartland. Saint Francis Hospice, Romford, UK

\subsection{6/bmjspcare-2017-hospice.255}

Background The Care Quality Commission requires hospices to evidence how views of service users are sought to inform and develop quality of care. Getting constructive comments is a challenge and can mean a gap in knowledge and understanding.

Aim To gather greater depth on service users' views of the care, using a focus group approach, to generate constructive feedback.

To establish a platform for listening, taking action and informing improvements.

Method Participants were recruited from two support groups and were diverse in age, gender and ethnic origin. All had experienced hospice care. 'You said we did' was emphasised to clarify the purpose. Two key questions guided discussion. Comments were organised into themes of 'What we do well' and 'What can we improve'. Additionally three participants undertook a video recorded interview in an adapted London taxi, provided by Health Watch Essex.

Findings Bereavement support and involvement with the hospice was important to many. Who accessed bereavement services was questioned. Support groups at evenings and weekends were requested. A range of approaches to care and support is needed as one size does not fit all. Extending the opening hours of the hospice shop elicited strong feelings; limited hours had a big impact. Some referral routes were difficult and this caused stress. The video material offers powerful stories.

Conclusion The focus group itself had a big impact on the attendees; being asked for their opinion was highly valued. Some feedback was constructive although further work is needed. Another focus group is planned for October 2017 to feedback on improvements made. The potential for learning from the service users is huge and goes beyond making specific improvements. Funding has been identified to increase bereavement support services. An outcome of the event is recruitment of 12 'Cloud Surfers' who will assist with reviewing marketing material.

\section{P-231 CREATING OPPORTUNITIES TO EXTEND PATIENT AND CARER INVOLVEMENT IN HOSPICE WORK}

Veronica Mickleburgh. LOROS Hospice, Leicester, UK

10.1136/bmjspcare-2017-hospice.256

Background Our current patient and carer participation group [PCPG] actively engages members with shaping the direction of the hospice whilst simultaneously improving the clinical environment and services.

Aim To create meaningful opportunities for our PCPG members to positively influence our services.

Methods Our three key opportunities are:

- Speaking out - members were invited to explore how we could use their experience in speaking out at LOROS events and to support campaigns involving the local media. Training was provided

- Participating in recruitment of key positions at LOROS

- Increasing the return rate of a comment card scheme Tell us what you think. Once a month, working with the patient experience lead, members go into clinical areas to engage patients with the scheme.

Results Following training, we now have a programme to utilise their new skills and past experience. So far, our members have attended several events including our annual cathedral concert.

Quote from a PCPG member - 
'It was challenging, rewarding and gave me confidence to speak in public on very emotive topics.'

Within only two months of developing the guidelines, our members have aided the recruitment of a senior receptionist and chaplain. Our return rate is increasing and areas for improvement are being highlighted through this approach. For example, issues around volunteer transport and patient-staff communication have been raised enabling us to identify and rectify issues at a faster rate.

Conclusion It's important to think of new ways of involving patients and carers in influencing decisions because they have the voice of experience and are valuable assets in improving our services. We plan to continue to offer these opportunities and involve more of our patients and carers in our work.

\section{P-232 'PIC' YOUR PATIENT: DEVELOPMENT OF A MULTI- PROFESSIONAL HANDOVER TOOL}

${ }^{1}$ Mark Mills, ${ }^{1,2}$ Claire Robinson, 'Laura Chapman. 'Marie Curie Hospice, Liverpool, UK; ${ }^{2}$ Aintree University Hospitals Foundation NHS Trust, Liverpool, UK

\subsection{6/bmjspcare-2017-hospice.257}

Background In a 26-bedded hospice it was recognised that handovers were excessive and inefficient. A review of practice revealed that they were occurring over 15 times per day with different professions present at each one. The Multi-disciplinary team (MDT) felt frustrated, recognising that the handovers reduced time for direct patient contact but still did not ensure timely sharing of crucial information. Staff wanted a single, efficient MDT handover to identify key patient issues. Method Current available tools were reviewed including SBAR (Haig, Sutton \& Whittington, 2006) (Situation, Background, Assessment, and Recommendation) but were found to be unsuitable for the hospice environment. The PIC acronym was created to facilitate nursing presentation of each patient. This prompted a discussion of: Plan (Is the patient going anywhere today? Are we working towards getting them to their preferred place of care?); Involvement (Are they being seen by the appropriate MDT members?); Concerns (Are you concerned about the patient or their family?). The PIC tool was used at the newly launched 'Huddle' - a handover led by a senior nurse and designated consultant, with a representative from each discipline of the MDT occurring each weekday morning from 0900-0910.

Results Informal feedback from staff suggested that the Huddle and PIC tool enabled more organised and efficient discharges. It was useful to give a view of the complexity of the patients throughout the hospice and guide admissions. A formal survey of the MDT is being carried out, with results available by October 2017 .

Conclusion Huddle is reducing the number of handovers each day and increasing the productivity of the hospice. This has benefits for swift discharge and assessing the needs of patients with complex problems. An unintended benefit has been improved nursing confidence in their presenting skills.

\section{P-233 THE PERSPECTIVE OF HOSPICE CARE FROM PEOPLE WITH MULTIPLE SCLEROSIS, A QUALITATIVE RESEARCH STUDY}

${ }^{1}$ Emma Hodges, ${ }^{2}$ Sue Read, ${ }^{2}$ Sotirios Santatzoglou. ${ }^{1}$ St Giles Hospice, Lichfield, UK; ${ }^{2}$ Keele University, Newcastle under Lyme, UK

10.1136/bmjspcare-2017-hospice.258

The research aimed to understand the lived experience of people living with multiple sclerosis (MS), their perspectives of hospice care and particularly the supportive care group at St Giles. St Giles runs three MS Groups which are based around exercise and wellbeing and as the attendees make a donation they are self-sustaining financially. As hospices consider their role in rehabilitative palliative care and for people with a palliative illness not in the last 12 months of life this study aims to contribute to strategic thinking. The groups are a model of service provision for people living with potentially long term palliative conditions. The research aimed to inform future service developments for people with MS in addition to other people who may benefit from this type of service. Whilst the service itself has been evaluated, exploring attendees' perspective in more depth and whether 'hospice' was a barrier to access hadn't been done.

The research study in partnership with Keele University involved three focus groups with 20 people participating and the opportunity for follow up with a semi-structured interview, six interviews were undertaken. A thematic analysis was then completed.

The results suggested that 'hospice' was an initial barrier for a small number of attendees, however for most the opportunity to exercise with other people with MS was the 'hook' that encouraged people to join the group. The results demonstrated the importance for attendees to be with other MS patients there was a theme of acceptance and shared understanding. There was a theme of insufficient information being available following diagnosis and a sense of 'being left to get on with it'. There was an incredible sense of resilience and community. There is learning that contributes to future service developments for other groups but also some additional support for the MS group that would add value.

\section{P-234 GATEWAY - A PORTAL TO THE RIGHT CARE AT THE RIGHT TIME AND IN THE RIGHT PLACE}

Maria Kavanagh. St Richard's Hospice, Worcester, UK

\subsection{6/bmjspcare-2017-hospice.259}

Background St Richard's Hospice have instigated a 'Gateway Service' for patient and family referrals to the hospice. This new service is designed to enable all patients to choose the level of care and support from the most appropriate specialist palliative care professional at the most appropriate time.

Aims Gateway aims to support national agendas by providing a better use of resources both physical and financial and by 